\title{
A two base pair deletion in the PQBP1 gene is associated with microphthalmia, microcephaly, and mental retardation
}

\author{
Isabel Martínez-Garay ${ }^{1,2}$, Miguel Tomás ${ }^{3}$, Silvestre Oltra ${ }^{1}$, Juliane Ramser ${ }^{4}$, Maria D Moltó ${ }^{2}$, \\ Félix Prieto $^{1}$, Alfons Meindl ${ }^{4}$, Kerstin Kutsche ${ }^{5}$ and Francisco Martínez ${ }^{*}, 1$
}

${ }^{1}$ Unidad de Genética, Hospital Universitario La Fe, Valencia, Spain; ${ }^{2}$ Departamento de Genética, Universidad de Valencia, Valencia, Spain; ${ }^{3}$ Servicio de Pediatría, Hospital Sant Francesc de Borja, Gandia, Spain; ${ }^{4}$ Department of Obstetrics and Gynaecology, Technical University Munich, Germany; ${ }^{5}$ Institut für Humangenetik, Universitätsklinikum Hamburg-Eppendorf, Hamburg, Germany

$X$-linked mental retardation has been traditionally divided into syndromic (S-XLMR) and non-syndromic forms (NS-XLMR), although the borderlines between these phenotypes begin to vanish and mutations in a single gene, for example PQBP1, can cause S-XLMR as well as NS-XLMR. Here, we report two maternal cousins with an apparently X-linked phenotype of mental retardation (MR), microphthalmia, choroid coloboma, microcephaly, renal hypoplasia, and spastic paraplegia. By multipoint linkage analysis with markers spanning the entire $\mathrm{X}$-chromosome we mapped the disease locus to a $28-\mathrm{Mb}$ interval between $\mathrm{Xp11.4}$ and $\mathrm{Xq12}$, including the BCOR gene. A missense mutation in $B C O R$ was described in a family with Lenz microphthalmia syndrome, a phenotype showing substantial overlapping features with that described in the two cousins. However, no mutation in the BCOR gene was found in both patients. Subsequent mutation analysis of $P Q B P 1$, located within the delineated linkage interval in Xp11.23, revealed a 2-bp deletion, c.461_462delAG, that cosegregated with the disease. Notably, the same mutation is associated with the Hamel cerebropalatocardiac syndrome, another form of S-XLMR. Haplotype analysis suggests a germline mosaicism of the 2-bp deletion in the maternal grandmother of both affected individuals. In summary, our findings demonstrate for the first time that mutations in PQBP1 are associated with an S-XLMR phenotype including microphthalmia, thereby further extending the clinical spectrum of phenotypes associated with PQBP1 mutations.

European Journal of Human Genetics (2007) 15, 29-34. doi:10.1038/sj.ejhg.5201717; published online 11 October 2006

Keywords: $P Q B P 1$; polyglutamine binding protein 1; microphthalmia; mental retardation; germline mosaicism; frameshift mutation

\section{Introduction}

To date, more than $120 \mathrm{X}$-linked inherited disorders have been described in which mental retardation (MR) appears

*Correspondence: F Martínez, Unidad de Genética, Hospital Universitario La Fe, Av. Campanar, 21, 46009-Valencia, Spain.

Tel/Fax: + 3496 1973153;

E-mail: francisco@gva.es

Received 7 February 2006; revised 6 July 2006; accepted 27 July 2006; published online 11 October 2006 as the primary character or at least a major component of the disease phenotype. ${ }^{1} \mathrm{X}$-linked mental retardation has been originally classified into syndromic (S-XLMR) and non-syndromic (NS-XLMR), depending on whether the patients display additional physical, behavioural, or neurological symptoms, or if MR is the only clinical manifestation. ${ }^{2,3}$ Nevertheless, the boundaries between S-XLMR and NS-XLMR are not always as strict as initially suggested, and affected persons of various NS-XLMR (MRX) families may 
display additional symptoms that have been missed at their first examination. ${ }^{4}$ Moreover, mutations in some of the known XLMR genes are associated with both syndromic as well as non-syndromic XLMR forms, indicating allelism in NS- and S-XLMR. ${ }^{5,6}$ For example, mutations in $P Q B P 1$, encoding the polyglutamine binding protein 1, were identified in patients with non-syndromic MR as well as in patients with syndromic MR and X-linked inheritance, including Sutherland-Haan, Renpenning, GolabiIto-Hall, Hamel cerebropalatocardiac, and Proteus syndrome. $^{7-13}$ Mutations in this gene tend to cluster in a dinucleotide repeat region located in exon 4 , adding or removing 2-bp units and thus causing a disruption of the PQBP1 open reading frame. Here, we report the identification of the same two base pair deletion, previously found in the affected members with Hamel cerebropalatocardiac syndrome (HCPCS), ${ }^{7}$ in two cousins with a severe disease phenotype comprising MR, microphthalmia, choroid coloboma, microcephaly, renal hypoplasia, and spastic paraplegia. To our knowledge, this is the first description of a PQBP1 mutation associated with microphthalmia.

\section{Materials and methods Subjects}

The present family includes two maternally related patients, two obligate carrier females, and five unaffected males (Figure 1a). Patient III.1 is the first son of a healthy non-consanguineous couple and was 29 years old at the time of examination. He was born after a normal pregnancy. Currently, he shows severe psychomotor retardation. He needs assistance with eating, is unable to walk independently or speak, and emits only guttural sounds. He had never developed seizures. At examination, the occipitofrontal circumference (OFC) was $46.5 \mathrm{~cm}(-6$ $\mathrm{SD})$. He also presented microphthalmia of the right eye and choroid coloboma of the left eye, spastic diplegia, camptodactyly, and arachnodactyly; his ears are large, simple, and protruding (Figure 2a). Numerous laboratory tests including biochemical analyses (of haemoglobin, copper, ceruloplasmin, uric acid, etc.) turned out to be normal. Conventional cytogenetic analysis revealed a normal 46,XY karyotype. The presence of a FRAXA mutation, responsible for the Fragile $\mathrm{X}$ syndrome, was excluded.

Patient III.8 is a 4-year-old maternal cousin of III.1. After preterm delivery (38 weeks), the following signs or conditions were referred: birth weight $2.150 \mathrm{~g}(-2.4 \mathrm{SD})$, length $41.5 \mathrm{~cm}(-5 \mathrm{SD})$, microcephaly (OFC 28.7; $-4.5 \mathrm{SD})$, microphthalmic right eye, retinal coloboma of the left eye, hypoplastic and malpositioned left kidney, and acute pylonephritis. A cranial MRI revealed that both ocular globes were of symmetric morphology with a size difference of $2 \mathrm{~mm}$ in anteroposterior diameter. In addition, he showed other dysmorphic signs such as large simple and protruding ears and a bulbous nose with broad nasal bridge (Figure 2b). Peripheral blood chromosome analysis

a

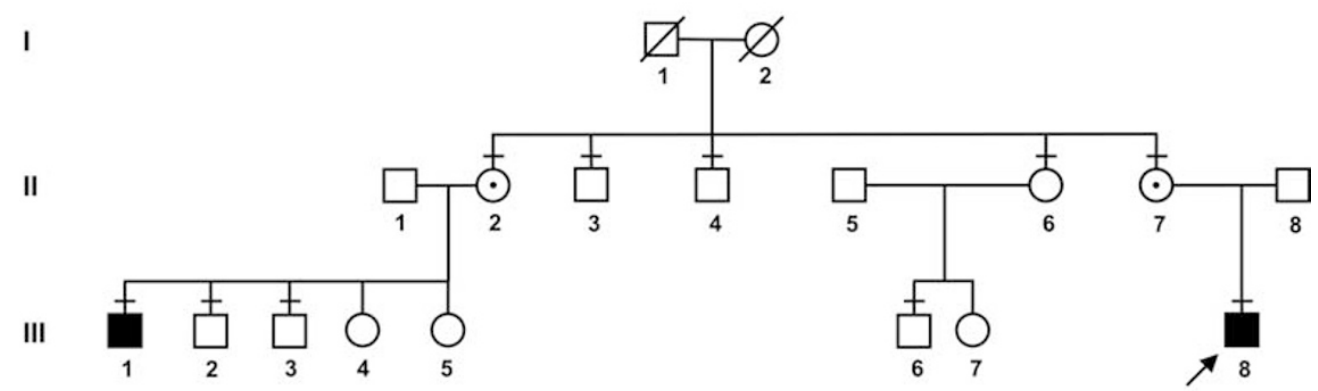

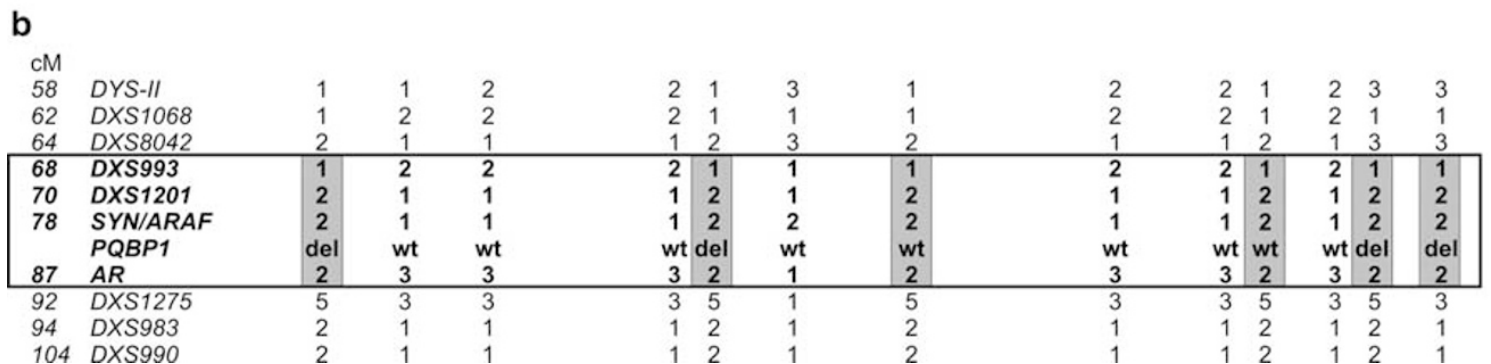

Figure 1 Pedigree of the family and haplotypes. (a) Affected males are indicated by blackened squares, whereas a dot indicates obligate carriers. The arrow points to the index patient. A minus (-) sign above a symbol indicates that a blood sample was obtained for analysis. (b) Haplotypes for markers in and around the linkage interval. The linkage interval is boxed. The disease-associated haplotype (grey background) is present in both affected males (III.1 and III.8), in the two obligate carrier females (II.2 and II.7), as well as in the unaffected maternal uncle (II.4), and the maternal aunt (II.6). The presence or absence of the dinucleotide deletion c.461_462delAG in PQBP1 is indicated (wt: wild-type; del: C.461_462delAG). 


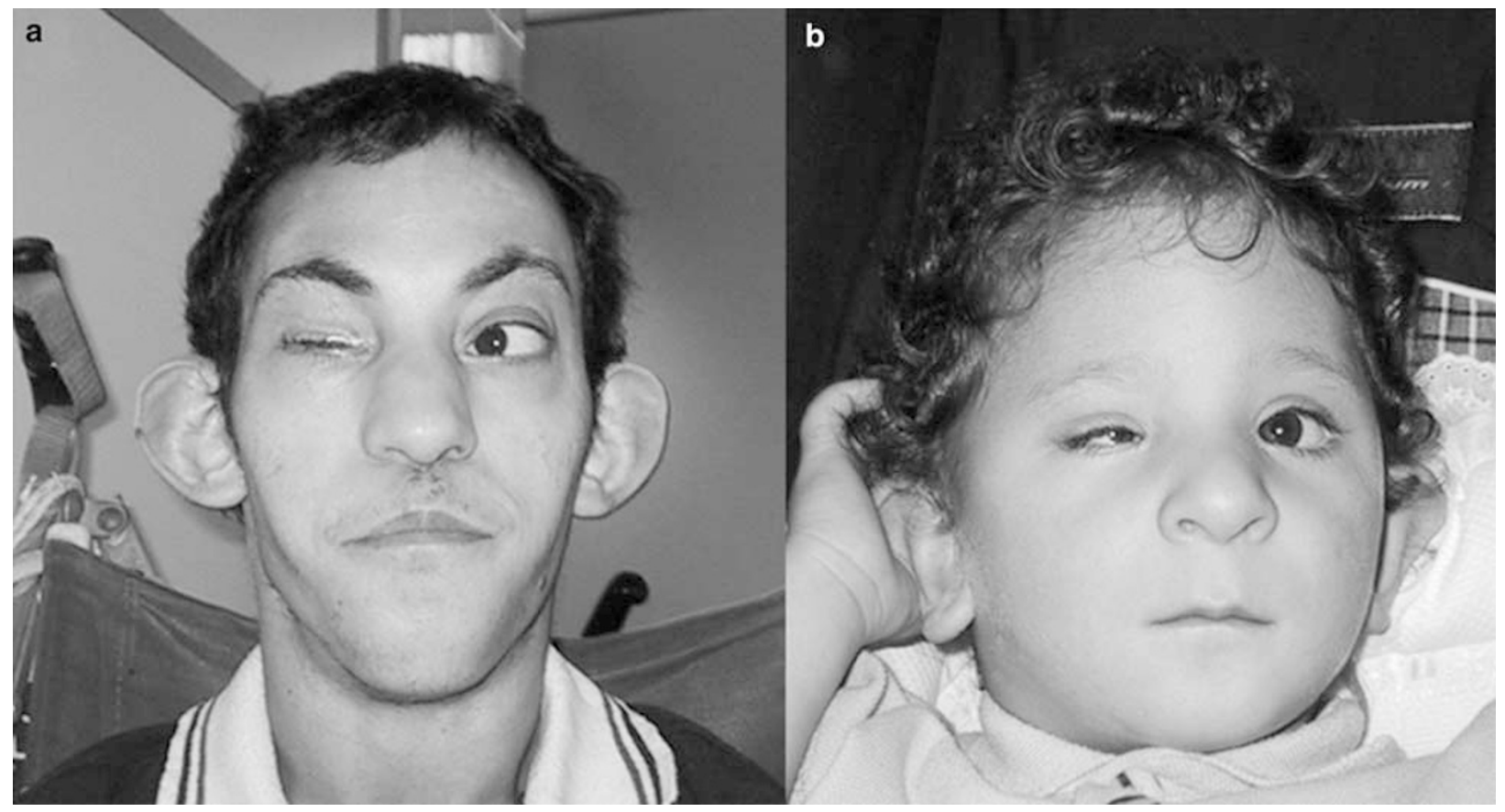

Figure 2 Photographs of the two patients. (a) Facial appearance of patient III.1 at the age of 29 years. Note microphthalmia of the right eye, long narrow face, microcephaly, large anteverted ears, bulbous nose, maxilla hypoplasia, and prognathism. (b) Facial view of patient III. 8 at the age of 4 years. He also shows microphthalmia of the right eye, microcephaly, and a bulbous nose. Note the rounded face at young age that has also been described for other patients with $P Q B P 1$ mutations.

revealed a normal 46,XY karyotype (at 550 band resolution). Under clinical examination, he showed developmental delay that is more pronounced at the motor area. The developmental quotient at 13 months of age showed the following scores: motor 58, coordination 53, language 65 and global 58 . At 21 months of age, he presented paroxysmal episodes consisting of atonia, blush face, and staring, which responded well to treatment. EEG study showed bilateral discharges of high-voltage spikes. At the age of 4 years, the patient was still unable to walk independently, he shows a very limited use of speech and multiple stereotypic movements of the hands. His weight was $12.5 \mathrm{~kg}(-2.6 \mathrm{SD})$, his length $96 \mathrm{~cm}(-2.3 \mathrm{SD})$, and his OFC $44 \mathrm{~cm}$ (-5.9SD). He shows large, low-set ears, high nasal root, micrognathia, long philtrum, and prominent metopic suture. Neurological symptoms include spastic diplegia with limited abduction angle and patellar hyperreflexia. Based on the above-mentioned clinical signs and symptoms, the diagnosis Lenz microphthalmia syndrome has been proposed. Informed consent was obtained from every member participating in the study.

\section{Genetic analysis}

DNA samples from various family members were isolated from blood samples by standard proteinase K/SDS lysis followed by phenol purification. Additionally, mononuclear cells were purified from peripheral blood of the patients under standard procedures, lysed in RLT (RNeasy kit, Qiagen) and immediately stored at $-70^{\circ} \mathrm{C}$. Total RNA was purified with the RNeasy kit (Qiagen), following the manufacturer's recommendations and then reverse transcribed into cDNA (RT-PCR Core Kit, PE Applied Biosystems). For linkage mapping, a total of 27 microsatellite markers spread along the entire X-chromosome were used. Lod scores for linkage between the disease locus and the genetic markers were calculated as described elsewhere. ${ }^{14}$

We amplified the coding region of BCOR (15 exons, GenBank accession no. AY316592) and PQBP1 (six exons, GenBank accession no. NM_005710), including the flanking intronic sequences, from genomic DNA and/or cDNA. Primer sequences and PCR conditions are available on request. PCR products were directly sequenced with the Big Dye Terminator ready reaction kit (PE Applied Biosystems) on an ABI-PRISM 3700 (PE Applied Biosystems).

\section{Results}

We performed multipoint linkage analysis using 22 informative X-chromosomal markers. The maximum Lod score, which hardly reached a score of 1.0, was obtained for marker DXS993 in Xp11.4 (data not shown). However, any interval outside the pericentromeric region could be excluded as only negative Lod values were obtained (below -2). After construction of the most likely segregating haplotypes, we found markers DXS993, DXS1201, SYN/ $A R A F$, and $A R$ to be tightly linked to the disease, whereas the closely linked markers DXS8042 and DXS1275 exhib- 
ited recombination (Figure $1 \mathrm{~b}$ ). These data indicated that the gene mutated in this family is located in Xp11.4-q12, in the region flanked by DXS8042 (distal) and DXS1275 (proximal). The disease-associated haplotype was shared by both affected patients III. 1 and III.8, their mothers (II.2 and II.7), as well as the maternal aunt (II.6), and, unexpectedly, the healthy maternal uncle (II.4) (Figure 1b). These data raised various possibilities to explain this phenomenon: first, the disease phenotype is inherited as an autosomalrecessive trait; second, the disease shows an incomplete penetrance; and third, the deceased maternal grandmother (I.2) carried a germline mosaicism. In view of these assumptions, it is of note that the phenotype of the two affected males resembles that of the Lenz microphthalmia syndrome, a syndromic form of X-linked MR, comprising microphthalmia/anophthalmia with MR, malformed ears, skeletal, renal, and urogenital abnormalities. ${ }^{15}$ Recently, a p.P85L missense mutation in the BCOR gene has been identified in males affected by Lenz microphthalmia in a single large family. ${ }^{16}$ Therefore, we performed mutation analysis of BCOR with genomic as well as cDNA of one affected person, but no sequence alteration was detected.

The PQBP1 gene, located in Xp11.23, was also present in the linkage interval of the family. This gene was considered a candidate as mutations in PQBP1 are associated with a clinical spectrum significantly overlapping with the phenotype of the described patients. ${ }^{5}$ Mutation screening of the six exons of $P Q B P 1$ revealed a dinucleotide deletion, c.461_462delAG, in both patients (III.1 and III.8) that has been previously described to be present in all affected males of the HCPCS family. ${ }^{7}$ This mutation could give rise to a truncated PQBP1 protein lacking 112 C-terminal amino acids, including part of the DR/ER repeat in the polar-amino-acid-rich domain as well as the nuclear localization signal and the C2 domain. Instead, 11 unrelated residues would be present at its C-terminus. The mothers of individuals III. 1 and III.8 (II. 2 and II.7) are heterozygous for the 2-bp deletion; however, the frameshift mutation was neither present in the unaffected maternal uncle (II.4) nor in the maternal aunt (II.6), who share the same haplotype. These data suggest that the dinucleotide deletion most likely occurred in germ cells of the deceased maternal grandmother (I.2), as the probability of two double recombinations is very low $(<0.00008)$.

In a previous study, we ascertained eight patients with proposed Lenz microphthalmia syndrome, nonetheless, mutation analysis of BCOR did not reveal any pathogenic mutation in either of them. ${ }^{17}$ Therefore, we searched for mutations in PQBP1 in these patients, but failed to detect a causative aberration.

\section{Discussion}

The frameshift mutation c.461_462delAG, identified in the two affected members of the present family, causes a premature stop codon that can either lead to a truncated PQBP1 protein or to a functional null-allele owing to nonsense-mediated mRNA decay. ${ }^{18}$ The same mutation was found in all affected males of the HCPCS family. Northern blot analysis of RNA isolated from lymphoblastoid cells of a patient from this family revealed almost no detectable $P Q B P 1$ transcript $^{7}$ indicating that the majority of PQBP1 mRNA is degraded owing to nonsense-mediated mRNA decay. These data suggest that the same pathogenetic mechanism can be applied to the 2-bp deletion present in this family.

The c.461_462delAG mutation gives rise to phenotypes that have numerous features in common, such as profound/severe MR, microcephaly, short stature, malar hypoplasia, spastic diplegia, long narrow face, bulbous nose, and large and protruding ears. ${ }^{8}$ However, the patients in the HCPCS family did not show any ocular and urogenital anomalies, including microphthalmia, coloboma, and renal hypoplasia (Table 1). Remarkably, mild skeletal abnormalities (clinodactyly of finger $V$ ) were observed in two patients with HCPCS, ${ }^{19}$ similarly, one of the two affected males presents with camptodactyly and arachnodactyly (Table 1). Spastic diplegia, present in both of our patients, has also been described for several other patients with $P Q B P 1$ mutations (Table 1). In general, the presence of malformations is not very common in males with PQBP1 mutations, although cardiac defects and cleft palate have been found in some cases. Although these data point to well recognizable phenotypes associated with PQBP1 mutations that should be unified under the name Renpenning syndrome, as suggested by Stevenson et al, ${ }^{11}$ additional features, for example severe eye abnormalities, can contribute to the high phenotypic variability of this syndromic form of X-linked MR. It has been suggested that differences in the truncated proteins and expression levels of the transcripts might be responsible for the more severe phenotype observed in the HCPCS family compared to other families associated with $P Q B P 1$ mutations. ${ }^{8}$ However, as the same mutation caused a more complex phenotype in the affected individuals of this family than in those of the HCPCS family, it is tempting to speculate that the high degree of the inter- and intra-familial variability is more likely due to yet unknown modifier genes, as already suggested for the phenotypes associated with NLGN4 mutations. ${ }^{20}$ In addition, epigenetic phenomena may similarly contribute to the development or repression of clinical manifestations.

The c.461_462delAG mutation was not present in an unaffected male and a female of this family, although they shared the disease-associated haplotype with both affected males as well as two obligate female carriers. The latter four individuals were found to carry the pathogenic PQBP1 mutation. These data indicate that the mutation most likely occurred in the germline of the maternal grandmother. Thus, the observation of a germline mosaicism in a 
Table 1 Clinical features of affected males with various mutations in PQBP1 as well as manifestations of patients with Lenz microphthalmia syndrome

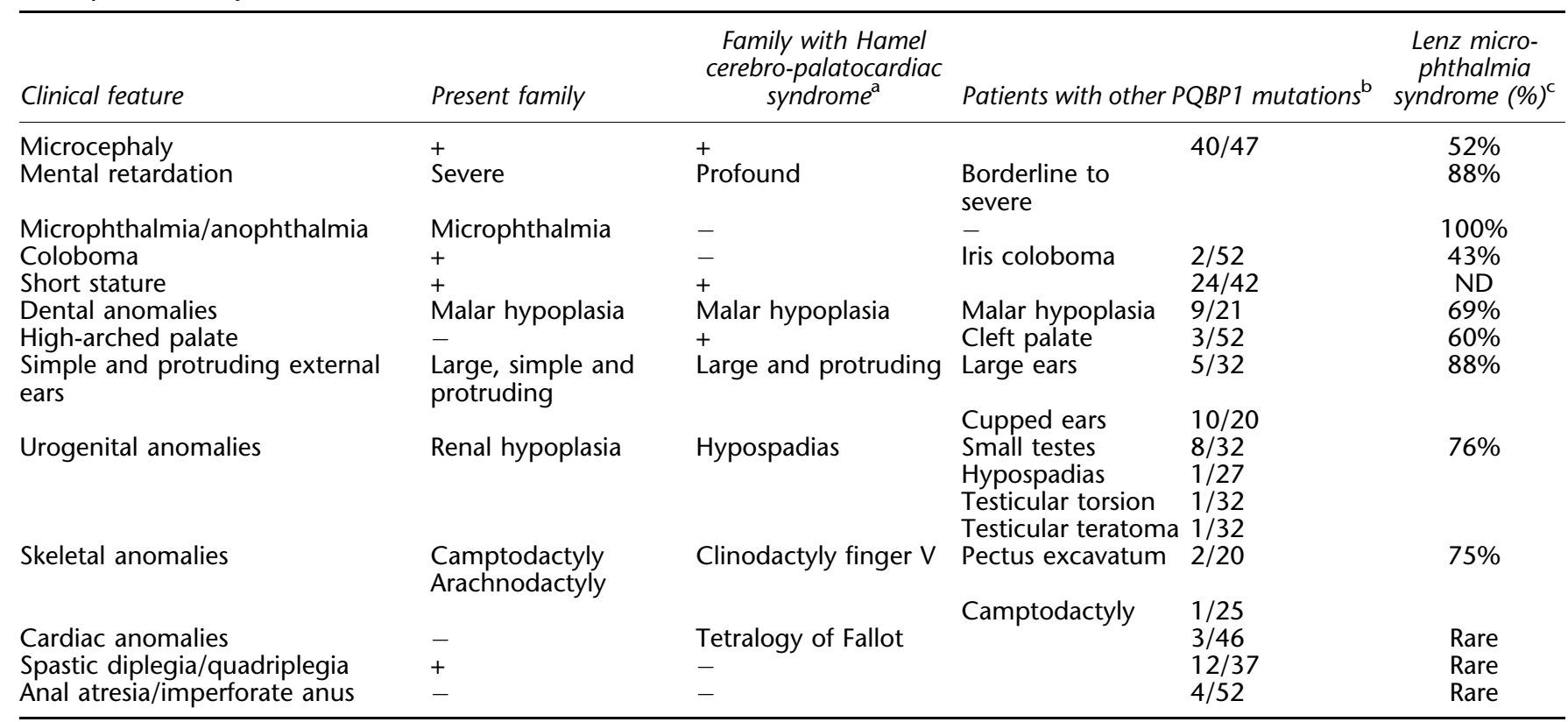

${ }^{a}$ Reported in Hamel et al ${ }^{19}$ and Kleefstra et al. ${ }^{8}$

${ }^{\mathrm{b}}$ Data reviewed in Kleefstra and Hamel ${ }^{5}$, Stevenson et $a l^{11}$, Fichera et $a l^{10}$, and Cossee et $a l^{13}$, without the Hamel cerebropalatocardiac family.

${ }^{C}$ Percentage of reported cases presenting the feature in Lenz microphthalmia syndrome (reviewed in $\mathrm{Ng}$ et al). ${ }^{27}$

ND, not determined.

female of this family adds the phenotypes associated with PQBP1 mutations to the growing list of X-linked inherited diseases in which germline mosaicism can be present, such as Lowe syndrome, ATR-X syndrome, Hunter syndrome, Duchenne muscular dystrophy, Rett syndrome, and haemophilia. ${ }^{21-24}$ Indeed, germline mosaicism is an important consideration for the interpretation of linkage data and should be taken into account in the counseling of parents of sporadic cases when the mutation is not detected in the mother.

The phenotype of the two affected males of this family was very similar to that of patients with Lenz microphthalmia syndrome (Table 1). Lenz microphthalmia syndrome is an X-linked recessive condition characterized by $\mathrm{MR}$, unilateral or bilateral microphthalmia, microcephaly, and urogenital, external ear, digital, and skeletal anomalies. ${ }^{15}$ Linkage analysis in two families with Lenz microphthalmia syndrome mapped the first locus associated with this disease to Xq27-q28 (MAA, MIM 309800). ${ }^{25}$ Recently, a single missense mutation (p.P85L) in $B C O R$ has been found to be associated with Lenz syndrome in another family, ${ }^{26}$ previously linked to Xp11.4 (MAA2, MIM 300412). ${ }^{16,27}$ However, no other causative $B C O R$ mutations have yet been documented in patients with Lenz syndrome. ${ }^{17}$ In eight patients with Lenz microphthalmia or related phenotypes, we did not find a pathogenic mutation in $P Q B P 1$. These data suggest that either $B C O R$ or $P Q B P 1$ are not the major genes for Lenz microphthalmia syndrome or a large genetic heterogeneity underlies this disorder.

In conclusion, the phenotype of the two affected males in this family shows substantial overlapping features with those described for other patients with PQBP1 mutations, demonstrating that these are allelic XLMR entities that could be combined under the name Renpenning syndrome. With the description of this family the phenotypic spectrum associated with PQBP1 mutations can be considerably extended, with MRX55 as the mildest form and this family presenting with the most severe phenotype.

\section{Acknowledgements}

We express our gratitude to the family for their active collaboration in the present study. We thank Inka Jantke for skillful technical assistance. This work was supported by Grants G03/098 and FISO4/ 0425 (Fondo de Investigación Sanitaria) from the Spanish Ministry of Health, as well as EC, QLG2-CT1999-00791 from the European Union.

\section{References}

1 Stevenson RE: Splitting and lumping in the nosology of XLMR. Am J Med Genet 2000; 97: 174-182.

2 Raymond FL: X-linked mental retardation: a clinical guide. J Med Genet 2006; 43: 193-200. 
3 Ropers HH, Hamel BC: X-linked mental retardation. Nat Rev Genet 2005; 6: 46-57.

4 Frints SG, Froyen G, Marynen P, Willekens D, Legius E, Fryns JP: Re-evaluation of MRX36 family after discovery of an ARX gene mutation reveals mild neurological features of Partington syndrome. Am J Med Genet 2002; 112: 427-428.

5 Kleefstra T, Hamel BC: X-linked mental retardation: further lumping, splitting and emerging phenotypes. Clin Genet 2005; 67: $451-467$.

6 Frints SG, Froyen G, Marynen P, Fryns JP: X-linked mental retardation: vanishing boundaries between non-specific (MRX) and syndromic (MRXS) forms. Clin Genet 2002; 62: 423-432.

7 Kalscheuer VM, Freude K, Musante L et al: Mutations in the polyglutamine binding protein 1 gene cause X-linked mental retardation. Nat Genet 2003; 35: 313-315.

8 Kleefstra T, Franken CE, Arens YH et al: Genotype-phenotype studies in three families with mutations in the polyglutaminebinding protein 1 gene (PQBP1). Clin Genet 2004; 66: 318-326.

9 Lenski C, Abidi F, Meindl A et al: Novel truncating mutations in the polyglutamine tract binding protein 1 gene (PQBP1) cause Renpenning syndrome and X-linked mental retardation in another family with microcephaly. Am J Hum Genet 2004; 74: 777-780.

10 Fichera M, Falco M, Lo Giudice $\mathrm{M}$ et al: Skewed X-inactivation in a family with mental retardation and PQBP1 gene mutation. Clin Genet 2005; 67: 446-447.

11 Stevenson RE, Bennett CW, Abidi F et al: Renpenning syndrome comes into focus. Am J Med Genet A 2005; 134: 415-421.

12 Abidi FE, Holloway L, Lubs H, Meindl A, Stevenson RE, Schwartz CE: Golabi-Ito-Hall syndrome results from a missense mutation in the WW domain of the PQBP1 gene. 54th Annual Meeting of the American Society of Human Genetics, Toronto, Canada, 2004.

13 Cossee M, Demeer B, Blanchet P et al: Exonic microdeletions in the X-linked PQBP1 gene in mentally retarded patients: a pathogenic mutation and in-frame deletions of uncertain effect. Eur J Hum Genet 2006; 14: 418-425.

14 Martinez F, Martinez-Garay I, Oltra S et al: Localization of MRX82: a new nonsyndromic X-linked mental retardation locus to Xq24q25 in a Basque family. Am J Med Genet A 2004; 131: 174-178.

15 Lenz W: [Recessive, sex-limited microphthalmia with multiple abnormalities]. Z Kinderheilkd 1955; 77: 384-390.
$16 \mathrm{Ng}$ D, Thakker N, Corcoran CM et al: Oculofaciocardiodental and Lenz microphthalmia syndromes result from distinct classes of mutations in BCOR. Nat Genet 2004; 36: 411-416.

17 Horn D, Chyrek M, Kleier S et al: Novel mutations in BCOR in three patients with oculo-facio-cardio-dental syndrome, but none in Lenz microphthalmia syndrome. Eur J Hum Genet 2005; 13: 563-569.

18 Lejeune F, Maquat LE: Mechanistic links between nonsensemediated mRNA decay and pre-mRNA splicing in mammalian cells. Curr Opin Cell Biol 2005; 17: 309-315.

19 Hamel BC, Mariman EC, van Beersum SE, Schoonbrood-Lenssen $\mathrm{AM}$, Ropers HH: Mental retardation, congenital heart defect, cleft palate, short stature, and facial anomalies: a new X-linked multiple congenital anomalies/mental retardation syndrome: clinical description and molecular studies. Am J Med Genet 1994; 51: 591-597.

20 Laumonnier F, Bonnet-Brilhault F, Gomot $\mathrm{M}$ et al: X-linked mental retardation and autism are associated with a mutation in the NLGN4 gene, a member of the neuroligin family. Am J Hum Genet 2004; 74: 552-557.

21 Bachoo S, Gibbons RJ: Germline and gonosomal mosaicism in the ATR-X syndrome. Eur J Hum Genet 1999; 7: 933-936.

22 Mari F, Caselli R, Russo S et al: Germline mosaicism in Rett syndrome identified by prenatal diagnosis. Clin Genet 2005; 67: 258-260.

23 Satre V, Monnier N, Berthoin $\mathrm{F}$ et al: Characterization of a germline mosaicism in families with Lowe syndrome, and identification of seven novel mutations in the OCRL1 gene. Am J Hum Genet 1999; 65: 68-76.

24 Zlotogora J: Germ line mosaicism. Hum Genet 1998; 102: $381-386$.

25 Forrester S, Kovach MJ, Reynolds NM, Urban R, Kimonis V: Manifestations in four males with and an obligate carrier of the Lenz microphthalmia syndrome. Am J Med Genet 2001; 98: 92-100.

26 Hoefnagel D, Keenan ME, Allen Jr FH: Heredofamilial bilateral anophthalmia. Arch Ophthalmol 1963; 69: 760-764.

$27 \mathrm{Ng}$ D, Hadley DW, Tifft CJ, Biesecker LG: Genetic heterogeneity of syndromic X-linked recessive microphthalmia-anophthalmia: is Lenz microphthalmia a single disorder? Am J Med Genet 2002; 110: $308-314$. 